Pacific

Journal of

Mathematics

\title{
THEORY OF NEWFORMS OF HALF-INTEGRAL WEIGHT
}

Murugesan Manickam, Jaban Meher

AND BALAKRISHNAN RAMAKRISHNAN 


\title{
THEORY OF NEWFORMS OF HALF-INTEGRAL WEIGHT
}

\author{
MuRUgeSAN MANiCKAM, JABAN MEHER \\ AND BALAKRISHNAN RAMAKRISHNAN
}

\begin{abstract}
We set up the theory of newforms of half-integral weight on $\Gamma_{0}(8 \mathrm{~N})$ and $\Gamma_{0}(16 N)$, where $N$ is odd and squarefree. Further, we extend the definition of the Kohnen plus space in general for trivial character and also study the theory of newforms in the plus spaces on $\Gamma_{0}(8 N), \Gamma_{0}(16 N)$, where $N$ is odd and squarefree. Finally, we show that the Atkin-Lehner $W$-operator $W_{4}$ acts as the identity operator on $S_{2 k}^{\text {new }}(4 N)$, where $N$ is odd and squarefree. This proves that $S_{2 k}^{-}(4)=S_{2 k}(4)$.
\end{abstract}

\section{Introduction}

Let $k, M$ be positive integers, $k \geq 2$. Write $M=2^{\alpha} N, \alpha \geq 0, N \geq 1, N$ odd. Let $\chi_{0}$ be a Dirichlet character modulo $N$ with $\epsilon=\chi_{0}(-1)$ and let $\chi_{1}$ be an even Dirichlet character modulo $2^{\alpha+2}$. Let $\chi=\left(\frac{4 \epsilon}{.}\right) \chi_{1} \chi_{0}$. Let $S_{k+1 / 2}(4 M, \chi)$ be the space of cusp forms of half-integral weight $k+\frac{1}{2}$ for $\Gamma_{0}(4 M)$ with character $\chi$, and let $S_{2 k}\left(2 M, \chi^{2}\right)$ be the space of cusp forms of weight $2 k$, level $2 M$ with character $\chi^{2}$. By the work of G. Shimura [1973] and S. Niwa [1975], there exist linear operators $\mathscr{S}_{t, 4 M, \chi}$ indexed by squarefree integers $t, \epsilon(-1)^{k} t>0$, which commute with the action of Hecke operators $T\left(n^{2}\right),(n, 2 M)=1$, and map the space $S_{k+1 / 2}(4 M, \chi)$ into the space $S_{2 k}\left(2 M, \chi^{2}\right)$. If $M$ is an odd integer, W. Kohnen [1980; 1982] introduced a canonical subspace $S_{k+1 / 2}^{+}(4 M, \chi)$, called the Kohnen plus space, in the full space $S_{k+1 / 2}(4 M, \chi)$. He defined modified Shimura lifts $\mathscr{S}_{D, 4 M, \chi}^{+}$, called Shimura-Kohnen lifts, indexed by fundamental discriminants $D, \epsilon(-1)^{k} D>0$, which commute with the action of Hecke operators $T\left(n^{2}\right)$, $(n, M)=1$, where $T(4)=T^{+}(4)$ is the Hecke operator introduced by Kohnen on the plus space. He proved that the linear operator $\mathscr{S}_{D, 4 M, \chi}^{+}$maps the space $S_{k+1 / 2}^{+}(4 M, \chi)$ into the space $S_{2 k}\left(M, \chi^{2}\right)$. The idea of characterising the spaces of half-integral weight forms Hecke-equivalent to a fixed integral weight newform is important, and establishing Hecke equivariant isomorphisms via trace identities is certainly a powerful tool. These isomorphisms often give hints as to how to further decompose these eigenspaces to obtain multiplicity-one results. The first such work

MSC2010: primary $11 \mathrm{~F} 37$; secondary $11 \mathrm{~F} 11,11 \mathrm{~F} 32$.

Keywords: modular forms of half-integral weight, theory of newforms, Kohnen plus space. 
was by Kohnen, who achieved that goal by introducing the plus space, which we now wish to generalise. Kohnen [1980; 1982] initiated the study of the theory of newforms for the plus space $S_{k+1 / 2}^{+}(4 M, \chi)$ along the lines of Atkin and Lehner [1970], where $M$ is odd and squarefree and $\chi^{2}=1$. Using the trace identities proved by Niwa [1977], M. Manickam, B. Ramakrishnan and T. C. Vasudevan [Manickam et al. 1990] set up the theory of newforms for the full space $S_{k+1 / 2}(4 M, \chi)$, where $M$ is odd and squarefree and $\chi^{2}=1$. If $M$ is even and squarefree, this theory is known on the full space $S_{k+1 / 2}(4 M, \chi)$ by the work of Manickam [1980; 2011]. For similar theories we refer to [Serre and Stark 1977; Shemanske 1996; Ueda 1988; 1991; 1993; 1998; 2001].

Kohnen introduced the plus space in $S_{k+1 / 2}(4 M, \chi)$ when $M$ is odd by letting $S_{k+1 / 2}^{+}(4 M, \chi)=\left\{f \in S_{k+1 / 2}(4 M, \chi): a_{f}(n)=0\right.$ unless $\left.\epsilon(-1)^{k} n \equiv 0,1(\bmod 4)\right\}$.

Ueda and Yamana [2010] extended the definition of the plus space for $S_{k+1 / 2}(4 M)$ ( $M$ is even and squarefree) by using the same condition on the Fourier coefficients. If $M$ is even, let

$$
S_{k+1 / 2}^{+}(4 M)=\left\{f \in S_{k+1 / 2}(4 M): a_{f}(n)=0 \text { unless }(-1)^{k} n \equiv 0,1(\bmod 4)\right\} .
$$

In the case where $M$ is odd, the Kohnen plus space $S_{k+1 / 2}^{+}(4 M)$ is an eigensubspace of $S_{k+1 / 2}(4 M)$ under a hermitian operator $U(4) W(4)$ [Kohnen 1982; Manickam et al. 1990], whereas in all other cases it is the image of the projection operator $\mathscr{P}_{+}$ on $S_{k+1 / 2}(4 M)$ ( $M$ even) given by

$$
\mathscr{P}_{+}: \sum_{n \geq 0} a(n) q^{n} \longrightarrow \sum_{\substack{n \geq 0 \\(-1)^{k} \\ n=0,1(\bmod 4)}} a(n) q^{n} .
$$

This operator $\mathscr{P}_{+}$was introduced by Kohnen and considered by Ueda and Yamana [2010]. If $M$ is even, $\mathscr{P}_{+}$preserves the space $S_{k+1 / 2}(4 M)$. This phenomenon is striking and it allows us to define the plus space for an even integer $M$ by

$$
S_{k+1 / 2}^{+}(4 M)=S_{k+1 / 2}(4 M) \mid \mathscr{P}_{+} .
$$

In this paper we generalise the theory of newforms for the Kohnen plus space and the full space whenever the traces of Hecke operators acting on the spaces of integral and half-integral weight modular forms are equal. We also consider the space $S_{k+1 / 2}(16 N), N$ odd and squarefree, and develop the theory of newforms by computing the dimension, since Ueda's trace formula is known for the case where the character of the space is nontrivial. In this case, we prove that the newform spaces $S_{k+1 / 2}^{\text {new }}(16 N)$ and $S_{k+1 / 2}^{+, \text {new }}(16 N)$ contain only the zero function.

Let us now explain the results of this paper. Let $M=2^{\alpha} N, \alpha=1,2, N$ odd and squarefree, $\chi^{2}=1$ and $\chi=\chi_{8}$ when $\alpha=2$, where $\chi_{8}$ is the real quadratic primitive 
even character modulo 8 defined by $\chi_{8}(n)=\left(\frac{2}{n}\right)$. Then there is a Hecke-equivariant isomorphism [Ueda 1988]

$$
\psi: S_{k+1 / 2}(4 M, \chi) \longrightarrow S_{2 k}(2 M) .
$$

We define the space of newforms in the full space as

$$
S_{k+1 / 2}^{\text {new }}(4 M, \chi)=\bigoplus_{F} S_{k+1 / 2}^{\text {new }}(4 M, \chi ; F),
$$

where the sum varies over an orthogonal basis of normalised Hecke eigenforms in $S_{2 k}^{\text {new }}(2 M)$, and for each such $F$ let

$S_{k+1 / 2}^{\text {new }}(4 M ; F)=\left\{f \in S_{k+1 / 2}(4 M, \chi): f \mid T\left(n^{2}\right)=a_{F}(n) f, \forall n \geq 1,(n, 2 M)=1\right\}$.

Then, $S_{k+1 / 2}^{\text {new }}(4 M, \chi)$ is the inverse image of $S_{2 k}^{\text {new }}(2 M)$ under the isomorphism $\psi$, so the "multiplicity-one" result is valid for $S_{k+1 / 2}^{\text {new }}(4 M, \chi)$.

Consider the plus space $S_{k+1 / 2}^{+}(8 N)$. Since $\mathscr{P}_{+}$preserves the space $S_{k+1 / 2}(8 N)$ and $\mathscr{P}_{+} T\left(n^{2}\right)=T\left(n^{2}\right) \mathscr{P}_{+},(n, 2 N)=1$, we define $S_{k+1 / 2}^{+, \text {new }}(8 N)=S_{k+1 / 2}^{\text {new }}(8 N) \mid \mathscr{P}_{+}$, and as such the plus space $S_{k+1 / 2}^{+, \text {new }}(8 N)$ is a subspace of $S_{k+1 / 2}^{\text {new }}(8 N)$. For a nonzero Hecke eigenform $f \in S_{k+1 / 2}^{\text {new }}(8 N ; F)$, the form $f \mid \mathscr{P}_{+}$is also a nonzero Hecke eigenform belonging to the same space having the same eigenvalues (for almost all Hecke operators) as that of $f$. Since $N$ is odd and squarefree, a multiplicityone result holds for the space $S_{k+1 / 2}^{\text {new }}(8 N)$ and hence $f \mid \mathscr{P}_{+}=f$. This proves the equality $S_{k+1 / 2}^{+, \text {new }}(8 N)=S_{k+1 / 2}^{\text {new }}(8 N)$. To get $f \mid \mathscr{P}_{+} \neq 0$, we use the multiplicity-one result along with the fact that $F \mid \mathscr{S}_{t}^{*} \neq 0$ for some squarefree integer $t \equiv 1(\bmod 4)$, $(-1)^{k} t>0$. Here $\mathscr{S}_{t}^{*}$ is the Shintani lifting, which is the adjoint of the Shimura map $\mathscr{Y}_{t}$ with respect to the Petersson scalar product $\left(\mathscr{S}_{t}\right.$ maps $S_{k+1 / 2}(8 N)$ into $\left.S_{2 k}(4 N)\right)$ - see [Manickam et al. 1989; Shintani 1975]. The nonvanishing of $F \mid \mathscr{S}_{t}^{*}$ follows from the fact that the $|t|$-th Fourier coefficient of $F \mid \mathscr{Y}_{t}^{*}$ is (up to a nonzero constant) equal to the special value $L(F, t, k)$ and, for some choice of squarefree integer $t,(t, 2 N)=1$, this special value is nonzero - see [Murty and Murty 1997]. Thus, we get $F\left|\mathscr{S}_{t}^{*}\right| \mathscr{P}_{+} \neq 0$, since $t \equiv 1(\bmod 4)$.

Now, we let $M=4 N$ and $\chi$ be trivial. Through the dimension formula we observe that $S_{k+1 / 2}^{\text {new }}(16 N)=S_{k+1 / 2}^{+ \text {,new }}(16 N)=\{0\}$. Further, we develop the theory of newforms on $S_{k+1 / 2}(16 N, \chi)$, where $\chi$ is trivial or $\chi=\chi_{8}$. Thus, in this paper we consider the above assumptions on $M$ :

$$
M= \begin{cases}2 N & \chi \text { trivial, } \\ 4 N & \chi \text { trivial or } \chi=\chi_{8},\end{cases}
$$

with $N$ odd and squarefree, and set up the theory of newforms. We observe that the Shimura-Kohnen lifts map the space $S_{k+1 / 2}^{+ \text {,new }}(8 N)$ into the space $S_{2 k}^{\text {new }}(4 N)$ instead of $S_{2 k}^{\text {new }}(2 N)$. 
Finally, as an application of the theory of newforms of half-integral weight, we get explicit eigenvalues for the $W$-operators on $S_{2 k}(2 M)$ (see [Gun et al. 2010], for example). More precisely, if $M=2 N$ or $4 N$ ( $N$ odd and squarefree), and if $F \in S_{2 k}^{\text {new }}(2 M)$ is a normalised newform with associated newform $f \in S_{k+1 / 2}^{\text {new }}(4 M, \chi)(8 \mid$ cond $\chi$ if $M=4 N$ ), then we have

$$
f \mid w_{p}=\left(\frac{D}{p}\right) f
$$

for all $p \mid N$, where $D$ is a fundamental discriminant, $(-1)^{k} D>0,(D, M)=1$ with $a_{f}(|D|) \neq 0$. To get this, we use $f \mid w_{p}=\lambda_{p} f$ and the explicit Fourier expansion of $f \mid w_{p}$ (see [Kohnen 1982]). Thus, for $p|N, F| W_{p}=\left(\frac{D}{p}\right) F$. Now,

$$
L^{*}(F, D, s):=\left(\frac{2 \pi}{\sqrt{2 M}|D|}\right)^{-s} \Gamma(s) L(F, D, s)
$$

satisfies

$$
L^{*}(F, D, 2 k-s)=\left(\frac{D}{2 M}\right) \lambda_{2 M} L^{*}(F, D, s), \quad\left(\frac{D}{-1}\right)=(-1)^{k},
$$

where $\lambda_{2 M}$ is the product of eigenvalues of the various $W$-operators $W_{p^{\beta}}$,

$$
\beta= \begin{cases}\alpha+1 & \text { if } p=2, \\ 1 & \text { otherwise. }\end{cases}
$$

Using $\lambda_{p}=\left(\frac{D}{p}\right)$ for all primes $p \mid N$ in the above functional equation, we get $\left(\frac{D}{2^{\beta}}\right) \cdot \lambda_{2^{\beta}}=1$, since $L(F, D, k)$ is nonzero for some fundamental discriminant $D,(D, 2 N)=1$. From this we conclude that the eigenvalue of the $W$-operator $W_{2^{\beta}}$ on $S_{2 k}^{\text {new }}(2 M)$ is equal to 1 when $\beta$ is even. This proves that $S_{2 k}(4)=S_{2 k}^{-}(4)$, where

$$
S_{2 k}^{-}(m)=\left\{f \in S_{2 k}(m): f \mid\left(\begin{array}{cc}
0 & -1 \\
m & 0
\end{array}\right)=f\right\} .
$$

The above subspace was introduced by Skoruppa and Zagier [1988] in connection with the theory of newforms for the space of Jacobi cusp forms.

\section{Preliminaries}

We begin by recalling some basic facts regarding modular forms of half-integral weight. Let $\mathscr{H}$ denote the upper half-plane consisting of complex numbers $\tau \in \mathbb{C}$ with $\operatorname{Im}(\tau)>0$. For complex numbers $z \neq 0, x$, we let $z^{x}=e^{x \log z}, \log z=\log |z|+i \arg z$, $-\pi<\arg z \leq \pi$. Let $\zeta$ be a fourth root of unity. Let $G$ denote the four-sheeted covering of $G L_{2}^{+}(\mathbb{Q})$ defined as the set of all ordered pairs $(\alpha, \phi(\tau))$, where $\phi(\tau)$ is a holomorphic function on $\mathscr{H}$ such that $\phi^{2}(\tau)=\zeta^{2}(c \tau+d) / \sqrt{\operatorname{det} \alpha}$ and $\alpha=\left(\begin{array}{ll}a & b \\ c & d\end{array}\right) \in G L_{2}^{+}(\mathbb{Q})$. Then $G$ is a group with multiplication $(\alpha, \phi(\tau))(\beta, \psi(\tau))=$ $(\alpha \beta, \phi(\beta \tau) \psi(\tau))$. Let $k \geq 2$ be a natural number. For a complex valued function $f$ defined on the upper half-plane $\mathscr{H}$ and an element $(\alpha, \phi(\tau)) \in G$, define the stroke 
operator by $\left.f\right|_{k+1 / 2}(\alpha, \phi(\tau))(\tau)=\phi(\tau)^{-2 k-1} f(\alpha \tau)$. We omit the subscript $k+\frac{1}{2}$ wherever there is no ambiguity. For $\Gamma_{0}(4)$ and its subgroups, we take the lifting $\Gamma_{0}(4) \rightarrow G$ as the collection $\{(\alpha, j(\alpha, \tau))\}$, where

$$
\alpha=\left(\begin{array}{ll}
a & b \\
c & d
\end{array}\right) \in \Gamma_{0}(4) \quad \text { and } \quad j(\alpha, \tau)=\left(\frac{c}{d}\right)\left(\frac{-4}{d}\right)^{-1 / 2}(c \tau+d)^{1 / 2} .
$$

Here $\left(\frac{c}{d}\right)$ denotes the generalised quadratic residue symbol and $\left(\frac{-4}{d}\right)^{1 / 2}$ is equal to 1 or $i$ according as $d$ is 1 or 3 modulo 4 . Let $M$ be a natural number. A holomorphic function $f: \mathscr{H} \rightarrow \mathbb{C}$ is called a modular form of weight $k+\frac{1}{2}$ for $\Gamma_{0}(4 M)$ with character $\chi$ (modulo $4 M$ ) if $\left.f\right|_{k+1 / 2}(\gamma, j(\gamma, \tau))(\tau)=\chi(d) f(\tau)$ for all $\gamma=\left(\begin{array}{ll}a & b \\ c & d\end{array}\right) \in \Gamma_{0}(4 M)$ and $f$ is holomorphic at all the cusps of $\Gamma_{0}(4 M)$. If, further, it vanishes at all the cusps, then it is called a cusp form. The set of cusp forms defined as above forms a complex vector space denoted by $S_{k+1 / 2}(4 M, \chi)$. If $\chi$ is the trivial character, then the space is denoted by $S_{k+1 / 2}(4 M)$. We also denote by $S_{k}(M)$ the space of cusp forms of weight $k$ on $\Gamma_{0}(M)$ with trivial character. The Fourier expansion of a cusp form $f$ at the cusp infinity is usually written as $f(\tau)=\sum_{n \geq 1} a_{f}(n) q^{n}$, where $q=e^{2 \pi i \tau}$. For a prime $p$, the $p$-th Hecke operator on $S_{k+1 / 2}(4 M)$ is denoted by $T\left(p^{2}\right)$ if $p \nmid 2 M$ and $U\left(p^{2}\right)$ if $p \mid 2 M$; and on $S_{2 k}(M)$ is denoted by $T(p)$ if $p \nmid M$ and $U(p)$ if $p \mid M$. By a Hecke eigenform in $S_{k+1 / 2}(4 M, \chi)$, we mean a nonzero form in the space which is a simultaneous eigenform for all Hecke operators $T\left(n^{2}\right),(n, 2 M)=1$. For any positive integer $n$, the operators $U(n)$ and $B(n)$ are defined on formal sums by $U(n): \sum_{m \geq 1} a(m) q^{m} \mapsto \sum_{m \geq 1} a(m n) q^{m}$, $B(n): \sum_{m \geq 1} a(m) q^{m} \mapsto \sum_{m \geq 1} a(m) q^{n m}$. The Petersson inner product for forms $f, g \in S_{k+1 / 2}(4 M)$ is defined by

$$
\langle f, g\rangle=\frac{1}{i_{4 M}} \int_{\mathscr{F}} f(\tau) \overline{g(\tau)} v^{k-3 / 2} d u d v,
$$

where $\mathscr{F}$ is a fundamental domain for the action of $\Gamma_{0}(4 M)$ on $\mathscr{H}, i_{4 M}$ is the index of $\Gamma_{0}(4 M)$ in $\mathrm{SL}_{2}(\mathbb{Z})$ and $\tau=u+i v$.

2.1. Shimura and Shintani liftings. Let $t$ be a squarefree integer with $(-1)^{k} t>0$. Then the $t$-th Shimura map on the space $S_{k+1 / 2}(4 M)$ is defined by

$$
f \mid \mathscr{S}_{t}=\sum_{n \geq 1}\left(\sum_{\substack{d \mid n \\(d, 2 M)=1}}\left(\frac{4 t}{d}\right) d^{k-1} a_{f}\left(|t| n^{2} / d^{2}\right)\right) q^{n} .
$$

We summarise the Shintani lifting [Manickam et al. 1989] when $M=2^{\alpha} N, N$ is odd and $\alpha \geq 1$. If $t$ is a squarefree integer, $(-1)^{k} t>0$, then for $F \in S_{2 k}(2 M)$ we have $F \mid \mathscr{S}_{t}^{*} \in S_{k+1 / 2}(4 M)$ and it is given by

$$
F \mid \mathscr{S}_{t}^{*}=(-1)^{[k / 2]} 2^{k-1+(a+1)(-k+1 / 2)} \sum_{m \geq 1}\left(\sum_{r \mid N} \mu(r)\left(\frac{t}{r}\right) r^{-k} r_{k, 2 M r}\left(F ; \Delta m r^{2}\right)\right) q^{m},
$$


where $r_{k, 2 M}(F ; \Delta m)$ is a certain cycle integral given by

$$
r_{k, 2 M}(F ; \Delta m)=\sum \omega_{t}(Q) \int_{C_{Q}} F(z)\left(a z^{2}+b z+c\right)^{k-1} d z .
$$

In the above, the sum is over all $\Gamma_{0}(2 M)$-equivalent quadratic forms $Q=[a, b, c]$ with discriminant $b^{2}-4 a c=\Delta m, \Delta=4^{\alpha+1}|t|$ and $a \equiv 0\left(\bmod 2^{2 \alpha+1} N\right) ; C_{Q}$ is the image in $\Gamma_{0}(2 M) \backslash \mathscr{H}$ of the semicircle $a|z|^{2}+b \Re(z)+c=0$ oriented from $(-b-\sqrt{\Delta} m) / 2 a$ to $(-b+\sqrt{\Delta} m) / 2 a$ if $a \neq 0$, or of the vertical line $b \Re(z)+c=0$ oriented from $-c / b$ to $i \infty$ if $b>0$ and from $i \infty$ to $-c / b$ if $b<0, a=0$.

Let us compute $r_{k, 2 M}(F ; \Delta|t|)$. Since $\Delta|t|=4^{\alpha+1} t^{2}$, we take the representatives $\left\{\left[0,2^{\alpha+1}|t|, \mu\right] \circ W_{r}: \mu\left(\bmod 2^{\alpha+1}|t|\right), r \mid 2 M, r>0\right\}$, where $W_{r}$ is the Atkin-Lehner $W$-operator. Note that $\omega_{t}\left(Q_{\mu} \circ W_{r}\right)=\left(\frac{t}{r}\right) \omega_{t}\left(Q_{\mu}\right)=\left(\frac{t}{r}\right)\left(\frac{t}{\mu}\right)$. Now, following the arguments in [Kohnen 1985, p. 243] we get

$$
r_{k, 2 M}\left(F ; 4^{\alpha+1} t^{2}\right)=2^{\nu(2 M)}(-1)^{[k / 2]}(2 \pi)^{-k} \Gamma(k)\left(2^{\alpha+1}|t|\right)^{k-1 / 2} L(F, t, k),
$$

where $v(2 M)$ is the number of prime factors of $2 M$. From this we get that, when $F$ is a newform, the $|t|$-th Fourier coefficient of $F \mid \mathscr{S}_{t}^{*}$ is (up to a nonzero constant) the special value $L(F, t, k)$.

2.2. W-operators and the projection operator $\mathscr{P}_{+}$. For $p \mid 2 N$, let $W_{p}$ denote the Atkin-Lehner $W$-operator on $S_{2 k}(2 N)$. For $p=2$, we define the analogous AtkinLehner $W$-operators $W(4)$ on $S_{k+1 / 2}(4 N)$ and $W(8)$ on $S_{k+1 / 2}(8 N)$ as follows:

$$
W(4)=\left(\left(\begin{array}{cc}
4 a & b \\
4 N c & 4
\end{array}\right), 2^{1 / 2} e^{i \pi / 4}(N c \tau+1)^{1 / 2}\right),
$$

where $a, b, c$ are integers satisfying $4 a-N b c=1$ and $b \equiv 1(\bmod 4)$;

$$
W(8)=\left(\left(\begin{array}{cc}
8 x & y \\
8 N w & 8
\end{array}\right), 8^{1 / 4} e^{i \pi / 4}(N w \tau+1)^{1 / 2}\right),
$$

where $x, y, w$ are integers such that $y \equiv 1(\bmod 8), 8 x-N w y=1$. We also let

$$
W_{*}(4)=\left(\left(\begin{array}{cc}
4 u & v \\
4 N r & 8
\end{array}\right), 2^{1 / 2} e^{i \pi / 4}(N r \tau+2)^{1 / 2}\right),
$$

where $r, u, v$ are integers satisfying $8 u-N r v=1$ and $v \equiv 1(\bmod 8)$.

Remark 2.1. The $W$-operators defined above are independent of the choice of the integers $a, b, c, x, y, w, r, u, v$ with the given conditions. We note that $W_{*}(4)=W(4)$ on $S_{k+1 / 2}(4 N)$; see [Manickam 1980; 2011] for details. The operator $W(8)$ maps $S_{k+1 / 2}(8 N)$ into $S_{k+1 / 2}\left(8 N, \chi_{8}\right)$, and $W(8)^{2}=I$ on $S_{k+1 / 2}(8 N, \chi)$, where $\chi$ is the principal character or $\chi=\chi_{8}$ and $I$ denotes the identity operator. 
We now define the projection operator $\mathscr{P}_{+}$on $S_{k+1 / 2}(4 M)$ when $M$ is even. Let $\xi=\left(\left(\begin{array}{ll}4 & 1 \\ 0 & 4\end{array}\right), e^{\pi i / 4}\right)$ and $\xi^{\prime}=\left(\left(\begin{array}{cc}4 & -1 \\ 0 & 4\end{array}\right), e^{-\pi i / 4}\right)$. Then a formal computation shows that $\xi$ (and hence $\xi^{\prime}$ ) preserves the space $S_{k+1 / 2}(4 M)$ if $4 \mid M$. Hence, if $4 \mid M$, we have

$$
\xi+\xi^{\prime}: S_{k+1 / 2}(4 M) \longrightarrow S_{k+1 / 2}(4 M) .
$$

However, in the following we prove the above property for any even integer $M$. Let $M=2 N$, where $N$ is an odd positive integer. We write

$$
\begin{aligned}
\xi+\xi^{\prime} & =\xi+\left(\begin{array}{cc}
1-2 N & (N-1) / 2 \\
8 N & 1-2 N
\end{array}\right)^{*} \xi\left(\begin{array}{cc}
1 & 0 \\
-8 N & 1
\end{array}\right)^{*} \\
& =\xi+\xi\left(\begin{array}{cc}
1 & 0 \\
-8 N & 1
\end{array}\right)^{*} \\
& =\xi \operatorname{Tr} \quad \text { on } S_{k+1 / 2}(8 N),
\end{aligned}
$$

where $\operatorname{Tr}=\sum_{\nu=0,1}\left(\begin{array}{cc}1 & 0 \\ -4 N \nu & 1\end{array}\right)^{*}$ is adjoint to the inclusion $S_{k+1 / 2}(8 N) \hookrightarrow S_{k+1 / 2}(16 N)$ with respect to the Petersson scalar product. On formal Fourier series $\sum a_{n} q^{n}$, we have

$$
\sum a_{n} q^{n} \mid\left(\xi+\xi^{\prime}\right)=\chi_{8}(2 k+1) \sqrt{2}\left(\sum_{(-1)^{k} n \equiv 0,1(\bmod 4)} a_{n} q^{n}-\sum_{(-1)^{k} n \equiv 2,3(\bmod 4)} a_{n} q^{n}\right) .
$$

We define

$$
\mathscr{P}_{+}:=\frac{1}{2}\left(\frac{\chi_{8}(2 k+1)}{\sqrt{2}}\left(\xi+\xi^{\prime}\right)+I\right)
$$

Then

$$
f \mid \mathscr{P}_{+}=\sum_{(-1)^{k} n \equiv 0,1(\bmod 4)} a_{f}(n) q^{n} \in S_{k+1 / 2}(4 M),
$$

where $f=\sum_{n \geq 1} a_{f}(n) q^{n} \in S_{k+1 / 2}(4 M)$.

\section{Newforms on the plus space $S_{k+1 / 2}^{+}(8 N)$}

In the recent work of Ueda and Yamana [2010], the plus space for $S_{k+1 / 2}(8 N)$ has been introduced and they studied the theory of newforms. In this case each newform in the full space $S_{k+1 / 2}^{\text {new }}(8 N)$ (see [Manickam 1980; 2011]) satisfies $f \mid \mathscr{P}_{+}=f$. This follows by using that $\mathscr{P}_{+}$maps $S_{k+1 / 2}^{\text {new }}(8 N)$ into itself and the multiplicity-one result obtained from Ueda's trace formula, together with the nonvanishing of $F \mid \mathscr{S}_{t}^{*}$ for some squarefree $t \equiv 1(\bmod 4)$, where $F \in S_{2 k}^{\text {new }}(4 N)$ is a normalised newform equivalent to $f$. Hence, the elements of $S_{k+1 / 2}^{\text {new }}(8 N)$ also satisfies the same plus space condition. Therefore, we consider the development of the theory of newforms on $S_{k+1 / 2}^{+}(8 N)$ and present the results in this section. 
Let us first state the results for the full space $S_{k+1 / 2}(8 N)$, where $N$ is odd and squarefree. The following orthogonal decomposition of $S_{k+1 / 2}(8 N)$ has been obtained in [Manickam 1980; 2011]:

$$
S_{k+1 / 2}(8 N)=S_{k+1 / 2}^{\text {new }}(8 N) \oplus S_{k+1 / 2}^{\text {old }}(8 N),
$$

where $S_{k+1 / 2}^{\text {new }}(8 N)=S_{k+1 / 2}^{+, \text {new }}(8 N)$ and the space of oldforms $S_{k+1 / 2}^{\text {old }}(8 N)$ has the decomposition

$$
\begin{gathered}
S_{k+1 / 2}^{\text {old }}(8 N)=\bigoplus_{r d \mid N, d<N} S_{k+1 / 2}^{+, \text {new }}(8 d)\left|U\left(r^{2}\right) \oplus \bigoplus_{r d \mid N} S_{k+1 / 2}^{\text {new }}(4 d)\right| U\left(r^{2}\right) \\
\bigoplus \bigoplus_{r d \mid N} S_{k+1 / 2}^{\text {new }}(4 d) U\left(r^{2}\right)\left|\mathscr{P}_{+} \oplus \bigoplus_{\substack{d|N \\
r d| 2 N}} S_{k+1 / 2}^{+, \text {new }}(4 d)\right| U\left(r^{2}\right) \\
\oplus \bigoplus_{r d \mid N} S_{k+1 / 2}^{+, \text {new }}(4 d)\left|U\left(4 r^{2}\right)\right| \mathscr{P}_{+} \cdot
\end{gathered}
$$

We need to show only that, for a fixed divisor $d \mid N$, the sum

$$
S_{k+1 / 2}^{+, \text {new }}(4 d)+S_{k+1 / 2}^{+, \text {new }}(4 d)\left|U(4)+S_{k+1 / 2}^{+, \text {new }}(4 d)\right| U(4) \mathscr{P}_{+}
$$

is direct. For some constants $\alpha, \beta, \gamma$ and a newform $f \in S_{k+1 / 2}^{+, \text {new }}(4 d)$, if we have

$$
\alpha f+\beta f|U(4)+\gamma f| U(4) \mathscr{P}_{+}=0,
$$

then, applying the operator $U(4)$ we get

$$
\alpha f|U(4)=-(\beta+\gamma) f| U(16),
$$

from which we conclude that $\alpha=0$. Since $S_{k+1 / 2}^{+, \text {new }}(4 d)\left|U(4) \oplus S_{k+1 / 2}^{+, \text {new }}(4 d)\right| U(4) \mathscr{P}_{+}$ is a direct sum, it follows that $\beta=\gamma=0$. This proves the required direct sum.

Thus, we get the following theorem regarding the plus space $S_{k+1 / 2}^{+}(8 N)$ :

Theorem 3.1. The plus space $S_{k+1 / 2}^{+}(8 N)$ has the orthogonal decomposition

where

$$
S_{k+1 / 2}^{+}(8 N)=S_{k+1 / 2}^{+, \text {new }}(8 N) \oplus S_{k+1 / 2}^{+, \text {old }}(8 N),
$$

$$
\begin{aligned}
S_{k+1 / 2}^{+, \text {old }}(8 N)=\bigoplus_{r d \mid N, d<N} S_{k+1 / 2}^{+, \text {new }}(8 d) \mid U\left(r^{2}\right) \oplus & \bigoplus_{r d \mid N} S_{k+1 / 2}^{\text {new }}(4 d) U\left(r^{2}\right) \mid \mathscr{P}_{+} \\
& \oplus \bigoplus_{r d \mid N} S_{k+1 / 2}^{+, \text {new }}(4 d)\left|U\left(4 r^{2}\right)\right| \mathscr{P}_{+}
\end{aligned}
$$

The spaces $S_{k+1 / 2}^{+, \text {new }}(8 N)$ and $S_{k+1 / 2}^{+, \text {old }}(8 N)$ are mapped into the spaces $S_{2 k}^{\text {new }}(4 N)$ and $S_{2 k}^{\text {old }}(4 N)$ respectively under the Shimura lifting. Moreover, the spaces of newforms $S_{k+1 / 2}^{+, \text {new }}(8 N)$ and $S_{2 k}^{\text {new }}(4 N)$ are isomorphic under a linear combination of Shimura lifts indexed by squarefree integers $t \equiv 1(\bmod 4),(-1)^{k} t>0$. 
Remark 3.2. If $f \in S_{k+1 / 2}^{+, \text {new }}(8 N)=S_{k+1 / 2}^{\text {new }}(8 N)$, then $a_{f}(n)=0$ whenever $(-1)^{k} n$ is not congruent to 1 modulo 4 . Hence, the Shimura maps $\mathscr{S}_{t, 8 N}$ annihilate $S_{k+1 / 2}^{\text {new }}(8 N)$ whenever $t \not \equiv 1(\bmod 4),(-1)^{k} t>0$.

\section{Newform theory on $S_{k+1 / 2}(16 N)$}

In this section, we extend the theory of newforms to the space $S_{k+1 / 2}(16 N)$, where $N$ is odd and squarefree. In this case, Ueda's trace formula is not valid as cond $\chi=1$. Also from the work of Manickam, Ramakrishnan and Vasudevan [Manickam et al. 1989] on the Shintani lifting, it seems that there exists no Shintani lift from $S_{2 k}^{\text {new }}(8 N)$ to $S_{k+1 / 2}(16 N)$. But, such a lifting exists if we replace the trivial character by a primitive character modulo 8 or 16 (see [Manickam et al. 1989]). This indicates the nonexistence of a nontrivial space of newforms in $S_{k+1 / 2}(16 N)$, which is mapped to $S_{2 k}^{\text {new }}(8 N)$ under the Shimura lifting. To realise this, we compute the dimension of the space $S_{k+1 / 2}(16 N)$ and give a decomposition of the space of oldforms (which turns out to be the full space).

Let us now compute the dimensions of the spaces $S_{2 k}(4 N)$ and $S_{k+1 / 2}(16 N)$. Using [Martin 2005], we have

$$
\begin{aligned}
\operatorname{dim} S_{2 k}(4 N) & =\frac{2 k-1}{12} 4 N \prod_{p \mid 2 N}\left(1+\frac{1}{p}\right)-\frac{3}{2} 2^{\nu(N)} \\
& =\frac{(2 k-1)}{2} \prod_{p \mid N}(p+1)-3 \cdot 2^{v(N)-1},
\end{aligned}
$$

where $v(N)$ is the number of prime factors of $N$. Now, using [Cohen and Oesterlé 1977], we get

$$
\begin{aligned}
\operatorname{dim} S_{k+1 / 2}(16 N) & =\frac{2 k-1}{24} 16 N \prod_{p \mid 2 N}\left(1+\frac{1}{p}\right)-\frac{\zeta(k, 16 N, 1)}{2} \prod_{p \mid N} \lambda\left(r_{p}, s_{p}, p\right) \\
& =(2 k-1) \prod_{p \mid N}(p+1)-3 \cdot 2^{v(N)} .
\end{aligned}
$$

(In the above we have used the dimension formula as given in [Ono 2004, Theorem 1.56, p. 16].) Equations (13), (14) imply that $\operatorname{dim} S_{k+1 / 2}(16 N)=2 \operatorname{dim} S_{2 k}(4 N)$.

We now state the main theorem of this section.

Theorem 4.1. We have

$$
S_{k+1 / 2}^{\text {new }}(16 N)=\{0\}
$$


(16)

$$
\begin{gathered}
S_{k+1 / 2}(16 N) \\
=\bigoplus_{r d \mid N}\left(S_{k+1 / 2}^{+, \text {new }}(4 d) \oplus S_{k+1 / 2}^{+, \text {new }}(4 d)\left|U(4) \oplus S_{k+1 / 2}^{+ \text {new }}(4 d)\right| U(4) \mathscr{P}_{+}\right. \\
\oplus S_{k+1 / 2}^{+, \text {new }}(4 d)\left|U(8) B(2) \oplus S_{k+1 / 2}^{+ \text {,new }}(4 d)\right| B(4) \\
\left.\oplus S_{k+1 / 2}^{+, \text {new }}(4 d) \mid U(4) B(4)\right) \mid U\left(r^{2}\right) \\
\oplus \bigoplus_{r d \mid N}\left(S_{k+1 / 2}^{\text {new }}(4 d) \oplus S_{k+1 / 2}^{\text {new }}(4 d)\left|P_{+} \oplus S_{k+1 / 2}^{\text {new }}(4 d)\right| U(2) B(2)\right. \\
\left.\oplus S_{k+1 / 2}^{\text {new }}(4 d) \mid B(4)\right) \mid U\left(r^{2}\right) \\
\oplus \bigoplus_{r d \mid N}\left(S_{k+1 / 2}^{\text {new }}(8 d) \oplus S_{k+1 / 2}^{\text {new }}(8 d) \mid W(16)\right) \mid U\left(r^{2}\right),
\end{gathered}
$$

where $W(16)$ is the $W$-operator corresponding to the prime $p=2$ in $S_{k+1 / 2}(16 N)$.

Proof. It is enough to show the direct sum in the respective eigensubspaces. First consider the eigensubspace generated by $S_{k+1 / 2}^{+ \text {,new }}(4 d)$. By Theorem 3.1, the sum $S_{k+1 / 2}^{+, \text {new }}(4 d)+S_{k+1 / 2}^{+ \text {,new }}(4 d)\left|U(4)+S_{k+1 / 2}^{+ \text {,new }}(4 d)\right| U(4) \mathscr{P}_{+}$is direct and, assuming the rest of the sum in the eigensubspace is not direct, then we have $f \in S_{k+1 / 2}^{+ \text {,new }}(4 d)$ which is nonzero and such that all odd coefficients of $f \mid U(8)$ are zero, by assuming $S_{k+1 / 2}^{+, \text {new }}(4 d) \mid U(8) \cap\left(S_{k+1 / 2}^{+, \text {new }}(4 d)\left|B(2)+S_{k+1 / 2}^{+ \text {, new }}(4 d)\right| U(4) B(2)\right)$ is nonzero. That is, $f \mid U(4) \in S_{k+1 / 2}(4 d)$ has the property that its $n$-th Fourier coefficient is zero whenever $n \equiv 2(\bmod 4)$. This means that $f \mid U(4) \in S_{k+1 / 2}^{+}(4 d)$, a contradiction since $0 \neq f \in S_{k+1 / 2}^{+}(4 d)$. Hence all the sums in the eigensubspace generated by $S_{k+1 / 2}^{+, \text {new }}(4 d)$ are direct. Next, consider the eigensubspace generated by $S_{k+1 / 2}^{\text {new }}(4 d)$. Clearly $S_{k+1 / 2}^{\text {new }}(4 d) \oplus S_{k+1 / 2}^{\text {new }}(4 d) \mid \mathscr{P}_{+}$is a direct sum in $S_{k+1 / 2}(8 N)$. If there is a nonzero element in the intersection of $S_{k+1 / 2}^{\text {new }}(4 d) \mid U(2) B(2)$ and $S_{k+1 / 2}^{\text {new }}(4 d) \mid B(4)$, then the $n$-th Fourier coefficient of a nonzero form $f \in S_{k+1 / 2}^{\text {new }}(4 d)$ vanishes whenever $n \equiv 2(\bmod 4)$ and hence, by [Kohnen 1982, Lemma], $0 \neq f \in S_{k+1 / 2}^{+}(4 d)$, a contradiction. So, the subspace $S_{k+1 / 2}\left|U(2) B(2) \oplus S_{k+1 / 2}^{\text {new }}(4 d)\right| B(4)$ is a direct sum in $S_{k+1 / 2}(16 N)$. In order to prove that all the sum as above generated by $S_{k+1 / 2}^{\text {new }}(4 d)$ is direct, we use the following fact. If $f \in S_{k+1 / 2}\left(8 N, \chi_{8}\right)$ and $f \mid B(2) \in S_{k+1 / 2}(8 N)$, then $f=0$, by [Serre and Stark 1977, Lemma 7]. Finally, applying $U(2)$ on the eigensubspace of $S_{k+1 / 2}^{\text {new }}(8 d)$, one component is mapped to zero and the other component is $S_{k+1 / 2}^{\text {new }}(8 d) \mid W(8)$, which is nonzero. Hence, we get that the sum in this eigensubspace is direct. This completes the proof for the direct sum decomposition of $S_{k+1 / 2}^{\text {old }}(16 N)$.

Since the spaces $S_{k+1 / 2}^{+, \text {new }}(4 d), S_{k+1 / 2}^{\text {new }}(4 d)$ and $S_{k+1 / 2}^{\text {new }}(8 d)$ are isomorphic (under the Shimura correspondence) to the spaces $S_{2 k}^{\text {new }}(d), S_{2 k}^{\text {new }}(2 d)$ and $S_{2 k}^{\text {new }}(4 d)$ respectively, we see that

$$
\operatorname{dim} S_{k+1 / 2}^{\text {old }}(16 N)=\sum_{r d \mid N}\left(6 \operatorname{dim} S_{2 k}^{\text {new }}(d)+4 \operatorname{dim} S_{2 k}^{\text {new }}(2 d)+2 \operatorname{dim} S_{2 k}^{\text {new }}(4 d)\right)
$$




$$
\begin{aligned}
& =2 \sum_{r d \mid N}\left(3 \operatorname{dim} S_{2 k}^{\text {new }}(d)+2 \operatorname{dim} S_{2 k}^{\text {new }}(2 d)+\operatorname{dim} S_{2 k}^{\text {new }}(4 d)\right) \\
& =2 \operatorname{dim} S_{2 k}(4 N)=\operatorname{dim} S_{k+1 / 2}(16 N)
\end{aligned}
$$

from the above computation. Therefore, it follows that $S_{k+1 / 2}^{\text {new }}(16 N)=\{0\}$.

\section{Newform theory on $S_{k+1 / 2}\left(16 N, \chi_{8}\right)$}

In this section, we study the theory of newforms on $S_{k+1 / 2}\left(16 N, \chi_{8}\right)$, where $\chi_{8}$ is the even quadratic character modulo 8 defined in the introduction and $N$ is odd and squarefree. Since cond $\chi_{8}=8$, by Ueda's result [1988] there exists a Hecke equivariant isomorphism between the spaces $S_{k+1 / 2}\left(16 N, \chi_{8}\right)$ and $S_{2 k}(8 N)$. Define the space of oldforms in $S_{k+1 / 2}\left(16 N, \chi_{8}\right)$ as follows:

$$
\begin{aligned}
& S_{k+1 / 2}^{\text {old }}\left(16 N, \chi_{8}\right) \\
& =\sum_{r d \mid N}\left(S_{k+1 / 2}^{+, \text {new }}(4 d)\left|B(2)+S_{k+1 / 2}^{+, \text {new }}(4 d)\right| U(2)\right) U\left(r^{2}\right) \\
& +\sum_{r d \mid N}\left(S_{k+1 / 2}^{+, \text {new }}(4 d)\left|U(8)+S_{k+1 / 2}^{+, \text {new }}(4 d)\right| U(8) W(8) B(2)\right) U\left(r^{2}\right) \\
& \quad+\sum_{r d \mid N}\left(S_{k+1 / 2}^{\text {new }}(4 d)\left|U(2)+S_{k+1 / 2}^{\text {new }}(4 d)\right| B(2)\right) U\left(r^{2}\right) \\
& \quad+\sum_{r d \mid N} S_{k+1 / 2}^{\text {new }}(4 d)\left|U(2) W(8) B(2) U\left(r^{2}\right)+\sum_{r d \mid N} S_{k+1 / 2}^{\text {new }}(8 d)\right| B(2) U\left(r^{2}\right) \\
& \quad+\sum_{r d \mid N} S_{k+1 / 2}^{\text {new }}(8 d)\left|W(8) U\left(r^{2}\right)+\sum_{r d \mid N, d<N} S_{k+1 / 2}^{\text {new }}\left(16 d, \chi_{8}\right)\right| U\left(r^{2}\right) .
\end{aligned}
$$

First consider the sum in the eigensubspace generated by $S_{k+1 / 2}^{+ \text {,new }}(4 d)$. Suppose $\left(f_{1} \mid U(4)+f_{2}\right)\left|U(2)=f_{3}\right| B(2)$, where $f_{i} \in S_{k+1 / 2}^{+, \text {new }}(4 d), i=1,2,3$. This implies that $f_{1} \mid U(4)+f_{2} \in S_{k+1 / 2}(4 d)$ is such that all its Fourier coefficients which are congruent to 2 modulo 4 are zero. Hence, by [Kohnen 1982, Lemma], we conclude that $f_{1} \mid U(4)+f_{2} \in S_{k+1 / 2}^{+}(4 d)$. Thus, $f_{1}=0$. Therefore, $f_{2}\left|U(2)=f_{3}\right| B(2)$, i.e., $f_{2}$ and $f_{2} \mid U(4)$ belong to $S_{k+1 / 2}^{+}(4 d)$, which implies that $f_{2}$ and hence $f_{3}=0$. Now, among the four components, the first three direct sums belong to $S_{k+1 / 2}\left(8 d, \chi_{8}\right)$. But, the fourth one is in $S_{k+1 / 2}(4 d) \mid B(2) \in S_{k+1 / 2}\left(16, \chi_{8}\right)$. This shows that all the four components form a direct sum. Next, consider the eigensubspaces generated by $S_{k+1 / 2}^{\text {new }}(4 d)$ and $S_{k+1 / 2}^{\text {new }}(8 d)$. A similar argument as above together with the following lemma shows that the respective sums are direct.

Lemma 5.1. The operator $U(2) W(8)$ has the following mapping property:

$$
U(2) W(8): S_{k+1 / 2}(4 N) \longrightarrow S_{k+1 / 2}(8 N) .
$$


Moreover, if $f \in S_{k+1 / 2}(4 N)$, then $f \mid U(2) W(8) \in S_{k+1 / 2}(4 N)$ if and only if $f \in S_{k+1 / 2}^{+}(4 N)$.

Proof. The mapping property follows from a straightforward verification. Suppose $f \mid U(2) W(8)=g$, where $f, g \in S_{k+1 / 2}(4 N)$. Using

$$
W(8) W_{*}(4)=\chi_{8}(2 k+1)\left(\left(\begin{array}{ll}
1 & 0 \\
0 & 2
\end{array}\right), 2^{1 / 4}\right) \quad \text { on } S_{k+1 / 2}\left(8 N, \chi_{8}\right)
$$

and

$$
W_{*}(4)=W(4) \text { on } S_{k+1 / 2}(4 N),
$$

we get

$$
f|U(2)|\left(\left(\begin{array}{ll}
1 & 0 \\
0 & 2
\end{array}\right), 2^{1 / 4}\right)=\chi_{8}(2 k+1) g \mid W(4) .
$$

Now, $g \mid W(4)$ is invariant under $\left(\begin{array}{ll}1 & 1 \\ 0 & 1\end{array}\right)^{*}$. Hence, $a_{f \mid U(2)}(n)=0$ if $n$ is odd and, therefore, $a_{f}(n)=0$ whenever $n \equiv 2(\bmod 4)$. This proves that $f \in S_{k+1 / 2}^{+}(4 N)$, a contradiction. For a detailed proof of the identities (19) and (20), we refer to [Manickam 2011].

Define the space of newforms in $S_{k+1 / 2}\left(16 N, \chi_{8}\right)$ to be the orthogonal complement (with respect to the Petersson scalar product) of $S_{k+1 / 2}^{\text {old }}\left(16 N, \chi_{8}\right)$ in $S_{k+1 / 2}\left(16 N, \chi_{8}\right)$. It is already known that the spaces $S_{k+1 / 2}^{+, \text {new }}(4 d), S_{k+1 / 2}^{\text {new }}(4 d)$ and $S_{k+1 / 2}^{\text {new }}(8 d)$ are isomorphic (respectively) to $S_{2 k}^{\text {new }}(d), S_{2 k}^{\text {new }}(2 d)$ and $S_{2 k}^{\text {new }}(4 d)$. Using induction on the number of prime factors of $N$, it follows that the space $S_{k+1 / 2}^{\text {new }}\left(16 d, \chi_{8}\right)$ is isomorphic to $S_{2 k}^{\text {new }}(8 d)$ if $d \mid N$ and $d<N$. Now, comparing the dimension of the space $S_{2 k}^{\text {old }}(8 N)$, we see that the spaces $S_{k+1 / 2}^{\text {old }}\left(16 N, \chi_{8}\right)$ and $S_{2 k}^{\text {old }}(8 N)$ have equal dimension. As mentioned at the beginning of this section, Ueda [1988] has shown that the spaces $S_{k+1 / 2}\left(16 N, \chi_{8}\right)$ and $S_{2 k}(8 N)$ are Heckeequivariantly isomorphic when $N$ is odd and squarefree. Therefore, combining all these facts, it follows that the space $S_{k+1 / 2}^{\text {new }}\left(16 N, \chi_{8}\right)$ is isomorphic to $S_{2 k}^{\text {new }}(8 N)$.

We summarise the results of this section in the following.

Theorem 5.2. Let $N$ be an odd and squarefree natural number and let $\chi_{8}$ be the primitive even quadratic Dirichlet character modulo 8 . Then $S_{k+1 / 2}\left(16 N, \chi_{8}\right)$ has an orthogonal decomposition

$$
S_{k+1 / 2}\left(16 N, \chi_{8}\right)=S_{k+1 / 2}^{\text {new }}\left(16 N, \chi_{8}\right) \oplus S_{k+1 / 2}^{\text {old }}\left(16 N, \chi_{8}\right),
$$


(21)

$$
\begin{aligned}
& S_{k+1 / 2}^{\text {old }}\left(16 N, \chi_{8}\right) \\
& =\bigoplus_{r d \mid N}\left(S_{k+1 / 2}^{+, \text {new }}(4 d)\left|B(2) \oplus S_{k+1 / 2}^{+, \text {new }}(4 d)\right| U(2) \oplus S_{k+1 / 2}^{+ \text {,new }}(4 d) \mid U(8)\right. \\
& \left.\oplus S_{k+1 / 2}^{+, \text {new }}(4 d) \mid U(8) W(8) B(2)\right) U\left(r^{2}\right) \\
& \oplus \bigoplus_{r d \mid N}\left(S_{k+1 / 2}^{\text {new }}(4 d)\left|U(2) \oplus S_{k+1 / 2}^{\text {new }}(4 d)\right| B(2)\right. \\
& \left.\bigoplus_{r d \mid N} S_{k+1 / 2}^{\text {new }}(4 d) \mid U(2) W(8) B(2)\right) U\left(r^{2}\right) \\
& \oplus \bigoplus_{r d \mid N}\left(S_{k+1 / 2}^{\text {new }}(8 d)\left|B(2) \oplus S_{k+1 / 2}^{\text {new }}(8 d)\right| W(8)\right) U\left(r^{2}\right) \\
& \oplus \bigoplus_{r d \mid N, d<N} S_{k+1 / 2}^{\text {new }}\left(16 d, \chi_{8}\right) \mid U\left(r^{2}\right) .
\end{aligned}
$$

The spaces $S_{k+1 / 2}^{\text {new }}\left(16 N, \chi_{8}\right)$ and $S_{k+1 / 2}^{\text {old }}\left(16 N, \chi_{8}\right)$ are mapped, respectively, into the spaces $S_{2 k}^{\text {new }}(8 N)$ and $S_{2 k}^{\text {old }}(8 N)$ under the Shimura lifting. Moreover, the spaces of newforms $S_{k+1 / 2}^{\text {new }}\left(16 N, \chi_{8}\right)$ and $S_{2 k}^{\text {new }}(8 N)$ are isomorphic under a linear combination of Shimura maps indexed by squarefree integers $t \equiv 1(\bmod 4),(-1)^{k} t>0$.

\section{Acknowledgements}

Some part of this work was done during Manickam and Ramakrishnan's visit to the Abdus Salam International Centre for Theoretical Physics, Trieste, Italy during May 2012. They thank the centre for the hospitality provided during their visit. Manickam also thanks the Harish-Chandra Research Institute, Allahabad for the hospitality during his visits to the institute. Finally, we thank the referee for a careful reading of the manuscript and for making useful suggestions.

\section{References}

[Atkin and Lehner 1970] A. O. L. Atkin and J. Lehner, "Hecke operators on $\Gamma_{0}(m)$ ", Math. Ann. 185 (1970), 134-160. MR 42 \#3022 Zbl 0177.34901

[Cohen and Oesterlé 1977] H. Cohen and J. Oesterlé, "Dimensions des espaces de formes modulaires", pp. 69-78 in Modular functions of one variable, VI (Bonn, 1976), edited by J.-P. Serre and D. B. Zagier, Lecture Notes in Math. 627, Springer, Berlin, 1977. MR 57 \#12396 Zbl 0371.10020

[Gun et al. 2010] S. Gun, M. Manickam, and B. Ramakrishnan, "A canonical subspace of modular forms of half-integral weight”, Math. Ann. 347:4 (2010), 899-916. MR 2011g:11088 Zbl 1219.11070

[Kohnen 1980] W. Kohnen, "Modular forms of half-integral weight on $\Gamma_{0}(4)$ ", Math. Ann. 248:3 (1980), 249-266. MR 81j:10030 Zbl 0416.10023

[Kohnen 1982] W. Kohnen, "Newforms of half-integral weight", J. Reine Angew. Math. 333 (1982), 32-72. MR 84b:10038 Zbl 0475.10025

[Kohnen 1985] W. Kohnen, "Fourier coefficients of modular forms of half-integral weight", Math. Ann. 271:2 (1985), 237-268. MR 86i:11018 Zbl 0542.10018

[Manickam 1980] M. Manickam, Newforms of half-integral weight and some problems on modular forms, thesis, University of Madras, 1980. 
[Manickam 2011] M. Manickam, "Newforms of half-integral weight on $\Gamma_{0}(8 N)$ ", pp. 63-71 in Number theory, edited by M. Manickam and B. Ramakrishnan, Ramanujan Math. Soc. Lect. Notes Ser. 15, Ramanujan Mathematical Society, Mysore, 2011. MR 2905488 Zbl 06103450

[Manickam et al. 1989] M. Manickam, B. Ramakrishnan, and T. C. Vasudevan, "On Shintani correspondence", Proc. Indian Acad. Sci. Math. Sci. 99:3 (1989), 235-247. MR 90m:11061 Zbl 0689.10033

[Manickam et al. 1990] M. Manickam, B. Ramakrishnan, and T. C. Vasudevan, "On the theory of newforms of half-integral weight", J. Number Theory 34:2 (1990), 210-224. MR 91b:11060 Zbl 0704.11013

[Martin 2005] G. Martin, "Dimensions of the spaces of cusp forms and newforms on $\Gamma_{0}(N)$ and $\Gamma_{1}(N)$ ", J. Number Theory 112:2 (2005), 298-331. MR 2005m:11069 Zbl 1095.11026

[Murty and Murty 1997] M. R. Murty and V. K. Murty, Non-vanishing of L-functions and applications, Progress in Mathematics 157, Birkhäuser, Basel, 1997. MR 98h:11106 Zbl 0916.11001

[Niwa 1975] S. Niwa, "Modular forms of half integral weight and the integral of certain thetafunctions", Nagoya Math. J. 56 (1975), 147-161. MR 51 \#361 Zbl 0303.10027

[Niwa 1977] S. Niwa, "On Shimura's trace formula", Nagoya Math. J. 66 (1977), 183-202. MR 58 \#27781 Zbl 0351.10018

[Ono 2004] K. Ono, The web of modularity: arithmetic of the coefficients of modular forms and $q$-series, CBMS Regional Conference Series in Mathematics 102, American Mathematical Society, Providence, RI, 2004. MR 2005c:11053 Zbl 1119.11026

[Serre and Stark 1977] J.-P. Serre and H. M. Stark, "Modular forms of weight 1/2", pp. 27-67 in Modular functions of one variable, VI (Bonn, 1976), edited by J.-P. Serre and D. B. Zagier, Lecture Notes in Math. 627, Springer, Berlin, 1977. MR 57 \#12400 Zbl 0371.10019

[Shemanske 1996] T. R. Shemanske, "Newforms of half-integral weight", Nagoya Math. J. 143 (1996), 147-169. MR 98k:11053 Zbl 0863.11032

[Shimura 1973] G. Shimura, "On modular forms of half integral weight", Ann. of Math. (2) 97 (1973), 440-481. MR 48 \#10989 Zbl 0266.10022

[Shintani 1975] T. Shintani, "On construction of holomorphic cusp forms of half integral weight", Nagoya Math. J. 58 (1975), 83-126. MR 52 \#10603 Zbl 0316.10016

[Skoruppa and Zagier 1988] N.-P. Skoruppa and D. B. Zagier, "Jacobi forms and a certain space of modular forms”, Invent. Math. 94:1 (1988), 113-146. MR 89k:11029 Zbl 0651.10020

[Ueda 1988] M. Ueda, "The decomposition of the spaces of cusp forms of half-integral weight and trace formula of Hecke operators", J. Math. Kyoto Univ. 28:3 (1988), 505-555. MR 90a:11054 Zbl 0673.10021

[Ueda 1991] M. Ueda, "The trace formulae of twisting operators on the spaces of cusp forms of half-integral weight and some trace relations", Japan. J. Math. (N.S.) 17:1 (1991), 83-135. MR 92g:11045 Zbl 0742.11031

[Ueda 1993] M. Ueda, "On twisting operators and newforms of half-integral weight", Nagoya Math. J. 131 (1993), 135-205. MR 95a:11040 Zbl 0778.11027

[Ueda 1998] M. Ueda, "On twisting operators and newforms of half-integral weight, II: Complete theory of newforms for Kohnen space", Nagoya Math. J. 149 (1998), 117-171. MR 99c:11050 Zbl 1016.11505

[Ueda 2001] M. Ueda, "On twisting operators and newforms of half-integral weight, III: Subspace corresponding to very-newforms", Comment. Math. Univ. St. Paul. 50:1 (2001), 1-27. MR 2002d:11048 Zbl 0995.11034 
[Ueda and Yamana 2010] M. Ueda and S. Yamana, "On newforms for Kohnen plus spaces", Math. Z. 264:1 (2010), 1-13. MR 2011a:11088 Zbl 1277.11045

Received April 24, 2014. Revised August 22, 2014.

MURUGESAN MANICKAM

KeRALA SCHOOL OF MATHEMATiCs

KUNNAMANGALAM P.O.

KOZHIKODE 673571

INDIA

murugumanick@ksom.res.in

JABAN MEHER

InSPIRE FELlow, DePARTMENT OF MATHEMATICS

INDIAN INSTITUTE OF SCIENCE

BANGALORE 560012

INDIA

jaban@math.iisc.ernet.in

BALAKRISHNAN RAMAKRISHNAN

HARISH-CHANDRA RESEARCH INSTITUTE

CHHATNAG RoAD, JHUNSI

AllahabAD 211019

INDIA

ramki@hri.res.in 


\title{
PACIFIC JOURNAL OF MATHEMATICS
}

\author{
msp.org/pjm
}

Founded in 1951 by E. F. Beckenbach (1906-1982) and F. Wolf (1904-1989)

\section{EDITORS}

Don Blasius (Managing Editor)

Department of Mathematics

University of California

Los Angeles, CA 90095-1555

blasius@math.ucla.edu

\author{
Paul Balmer \\ Department of Mathematics \\ University of California \\ Los Angeles, CA 90095-1555 \\ balmer@math.ucla.edu \\ Robert Finn \\ Department of Mathematics \\ Stanford University \\ Stanford, CA 94305-2125 \\ finn@math.stanford.edu \\ Sorin Popa \\ Department of Mathematics \\ University of California \\ Los Angeles, CA 90095-1555 \\ popa@math.ucla.edu
}

\author{
Vyjayanthi Chari \\ Department of Mathematics \\ University of California \\ Riverside, CA 92521-0135 \\ chari@math.ucr.edu \\ Kefeng Liu \\ Department of Mathematics \\ University of California \\ Los Angeles, CA 90095-1555 \\ liu@math.ucla.edu \\ Jie Qing \\ Department of Mathematics \\ University of California \\ Santa Cruz, CA 95064 \\ qing@ cats.ucsc.edu
}

\section{PRODUCTION}

Silvio Levy, Scientific Editor, production@msp.org

\section{SUPPORTING INSTITUTIONS}

ACADEMIA SINICA, TAIPEI

CALIFORNIA INST. OF TECHNOLOGY

INST. DE MATEMÁTICA PURA E APLICADA

KEIO UNIVERSITY

MATH. SCIENCES RESEARCH INSTITUTE

NEW MEXICO STATE UNIV.

OREGON STATE UNIV.

\author{
STANFORD UNIVERSITY \\ UNIV. OF BRITISH COLUMBIA \\ UNIV. OF CALIFORNIA, BERKELEY \\ UNIV. OF CALIFORNIA, DAVIS \\ UNIV. OF CALIFORNIA, LOS ANGELES \\ UNIV. OF CALIFORNIA, RIVERSIDE \\ UNIV. OF CALIFORNIA, SAN DIEGO \\ UNIV. OF CALIF., SANTA BARBARA
}

\author{
Daryl Cooper \\ Department of Mathematics \\ University of California \\ Santa Barbara, CA 93106-3080 \\ cooper@math.ucsb.edu \\ Jiang-Hua Lu \\ Department of Mathematics \\ The University of Hong Kong \\ Pokfulam Rd., Hong Kong \\ jhlu@maths.hku.hk \\ Paul Yang \\ Department of Mathematics \\ Princeton University \\ Princeton NJ 08544-1000 \\ yang@math.princeton.edu
}

These supporting institutions contribute to the cost of publication of this Journal, but they are not owners or publishers and have no responsibility for its contents or policies.

See inside back cover or msp.org/pjm for submission instructions.

The subscription price for 2015 is US \$420/year for the electronic version, and \$570/year for print and electronic.

Subscriptions, requests for back issues and changes of subscribers address should be sent to Pacific Journal of Mathematics, P.O. Box 4163, Berkeley, CA 94704-0163, U.S.A. The Pacific Journal of Mathematics is indexed by Mathematical Reviews, Zentralblatt MATH, PASCAL CNRS Index, Referativnyi Zhurnal, Current Mathematical Publications and Web of Knowledge (Science Citation Index).

The Pacific Journal of Mathematics (ISSN 0030-8730) at the University of California, c/o Department of Mathematics, 798 Evans Hall \#3840, Berkeley, CA 94720-3840, is published twelve times a year. Periodical rate postage paid at Berkeley, CA 94704, and additional mailing offices. POSTMASTER: send address changes to Pacific Journal of Mathematics, P.O. Box 4163, Berkeley, CA 94704-0163.

PJM peer review and production are managed by EditFLOW ${ }^{\circledR}$ from Mathematical Sciences Publishers.

\section{PUBLISHED BY}

\section{mathematical sciences publishers \\ nonprofit scientific publishing}

http://msp.org/

(C) 2015 Mathematical Sciences Publishers 


\section{PACIFIC JOURNAL OF MATHEMATICS}

Volume $274 \quad$ No. $1 \quad$ March 2015

Unimodal sequences and "strange" functions: a family of quantum modular forms

KATHRIN BRINGMANN, AMANDA FOLSOM and ROBERT C.

RHOADES

Congruence primes for Ikeda lifts and the Ikeda ideal

JIM BROWN and RODNEY KEATON

Constant mean curvature, flux conservation, and symmetry

NiCK EDELEN and BRUCE SOLOMON

The cylindrical contact homology of universally tight sutured contact solid tori

ROMAN GOLOVKO

Uniform boundedness of $S$-units in arithmetic dynamics

Holly Krieger, Aaron LeVin, Zachary Scherr, ThOMAS

TUCKER, Yu YASUFuKu and MiCHAEL E. ZiEVE

A counterexample to the energy identity for sequences of $\alpha$-harmonic 107 maps

YUXIANG LI and YOUDE WANG

Theory of newforms of half-integral weight

Murugesan MANiCKAM, JABAN MEHER and BALAKRISHNAN RAMAKRISHNAN

Algebraic families of hyperelliptic curves violating the Hasse principle

NGUYEN NGOC DONG QUAN

$F$-zips with additional structure

RichaRd PINK, TORSTEN WEDHORN and PAUL ZiEgler

Mean values of $L$-functions over function fields 